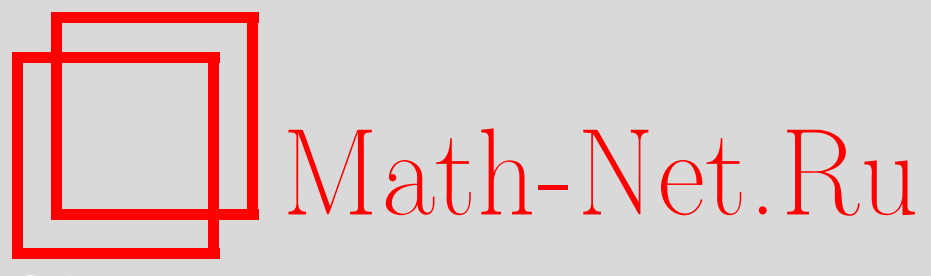

К. М. Эминян, Проблема Гольдбаха в простых числах с двоичными разложениями специального вида, Изв. РАН. Сер. матем., 2014, том 78, выпуск 1, 215-224

DOI: https://doi.org/10.4213/im7989

Использование Общероссийского математического портала Math-Net.Ru подразумевает, что вы прочитали и согласны с пользовательским соглашением http://www . mathnet.ru/rus/agreement

Параметры загрузки:

IP : 54.210 .77 .194

26 апреля 2023 г., 14:13:57

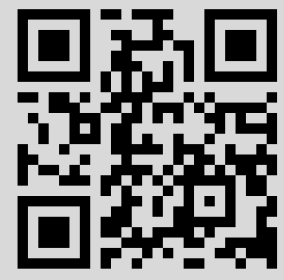


УДК 511

\section{К. М. Эминян \\ Проблема Гольдбаха в простых числах с двоичными разложениями специального вида}

Получена асимптотическая формула для числа представлений нечетного числа $N$ суммой трех простых чисел из множества $\mathbb{N}_{0}$, где $\mathbb{N}_{0}-$ множество натуральных чисел, двоичные разложения которых имеют четное число 1.

Библиография: 6 наименований.

Ключевые слова: тернарная проблема Гольдбаха, проблема Гельфонда, большое решето, тригонометрические суммы.

\section{$\S 1$. Введение}

Пусть $n=\varepsilon_{0}+\varepsilon_{1} 2+\cdots+\varepsilon_{k} 2^{k}-$ представление натурального числа $n$ в двоичной системе счисления, $\varepsilon_{j}=0,1$. Пусть

$$
\mathbb{N}_{0}=\left\{n: n \in \mathbb{N} ; \sum_{k=0}^{\infty} \varepsilon_{k} \equiv 0(\bmod 2)\right\}, \quad \mathbb{N}_{1}=\left\{n: n \in \mathbb{N} ; \sum_{k=0}^{\infty} \varepsilon_{k} \equiv 1(\bmod 2)\right\}
$$

и пусть

$$
\varepsilon(n)=\left\{\begin{array}{lll}
1, & \text { если } & n \in \mathbb{N}_{0}, \\
-1, & \text { если } & n \in \mathbb{N}_{1} .
\end{array}\right.
$$

А. О. Гельфонд в 1968 г. доказал [1], что числа классов $\mathbb{N}_{0}$ и $\mathbb{N}_{1}$ регулярно распределены в арифметических прогрессиях.

В 1991 г. автор получил [2] асимптотическую формулу для суммы

$$
\sum_{n \leqslant x, n \in \mathbb{N}_{0}} \tau(n)
$$

и тем самым решил проблему делителей Дирихле в числах класса $\mathbb{N}_{0}$.

K. Маудюит и Дж. Риват в 2010 г. доказали [3], в частности, что плотности множеств простых чисел из классов $\mathbb{N}_{0}$ и $\mathbb{N}_{1}$ совпадают. Другое доказательство этого факта дал Б. Грин [4]. Результаты, полученные в указанных работах, основаны на оценках специального вида тригонометрических сумм, которые как по своей силе, так и по доказательствам представляют собой варианты принадлежащей автору оценки интеграла от модуля тригонометрической суммы специального вида, полученной в [2, лемма 4].

В настоящей работе решается тернарная проблема Гольдбаха в простых числах из класса $\mathbb{N}_{0}$.

Основные результаты содержатся в следующих теоремах.

(C) К. М. Эминян, 2014 


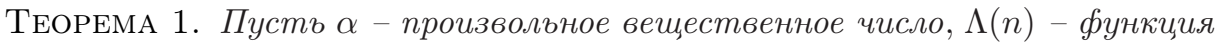
Мангольдта. Тогда существует абсолютная постоянная $\varkappa>0$ такая, что

$$
S=\sum_{n \leqslant X} \varepsilon(n) \Lambda(n) e^{2 \pi i \alpha n}=O\left(X^{1-\varkappa}\right) .
$$

Постоянная в знаке $O$ абсолютная.

Теорема 2. Пусть $J(N)$ - число представлений нечетного $N$ суммой трех простых чисел, и пусть $J_{0}(N)$ - число представлений нечетного $N$ суммой трех простьх чисел из класса $\mathbb{N}_{0}$. Тогда справедливо равенство

$$
J_{0}(N)=\frac{1}{8} J(N)\left(1+O\left(N^{-\varkappa} \ln N\right)\right),
$$

где $\varkappa>0$ - константа из теоремы 1.

\section{§ 2. Вспомогательные леммы}

ЛЕмма 1. Пусть $\alpha=\frac{a}{q}+\frac{\theta}{q^{2}},(a, q)=1, q \geqslant 1,|\theta| \leqslant 1$. Тогда при любом $\beta \in \mathbb{R} u U>0, P \geqslant 1$ имеем

$$
\sum_{x=1}^{P} \min \left(U,\|\alpha x+\beta\|^{-1}\right) \leqslant 6\left(\frac{P}{q}+1\right)(U+q \ln q) .
$$

ДОКАЗАТЕЛЬСТвО см. в [5, гл. 4].

Лемма 2 (А.О. Гельфонд). Пусть $Q \in \mathbb{N}$. Справедливо неравенство

$$
\left|\prod_{r=0}^{2 Q-1}\left(1-e^{2 \pi i \alpha 2^{r}}\right)\right| \leqslant \frac{2}{\sqrt{3}} 2^{2 Q \lambda}
$$

где $\lambda=\frac{\ln 3}{\ln 4}=0.7924812 \ldots$.

ДокАЗАТЕЛЬСтво см. в [1].

СлЕДСТВИЕ 1. При любом $\alpha \in \mathbb{R}$ справедлива оченка

$$
\left|\sum_{n \leqslant X} \varepsilon(n) e^{2 \pi i \alpha n}\right|=O\left(X^{\lambda} \ln X\right) .
$$

ДоказАтельство. Определим натуральное число $Q$ из неравенств

$$
2^{2(Q-1)}<X+1 \leqslant 2^{2 Q} .
$$

Тогда

$$
\begin{aligned}
\left|\sum_{n \leqslant X} \varepsilon(n) e^{2 \pi i \alpha n}\right| & =\left|\sum_{n<2^{2 Q}} \varepsilon(n) e^{2 \pi i \alpha n} \sum_{n_{1} \leqslant X} \frac{1}{2^{2 Q}} \sum_{l=1}^{2^{2 Q}} e^{2 \pi i\left(n-n_{1}\right) \cdot 2^{-2 Q} l}\right| \\
& \leqslant 2^{-2 Q} \sum_{l=1}^{2^{2 Q}}\left|\sum_{n<2^{2 Q}} \varepsilon(n) e^{2 \pi i\left(\alpha+2^{-2 Q} l\right) n}\right|\left|\sum_{n_{1} \leqslant X} e^{-2 \pi i n_{1} 2^{-2 Q} l}\right| .
\end{aligned}
$$


Далее, из тождества

$$
\sum_{n<2^{2 Q}} \varepsilon(n) e^{2 \pi i\left(\alpha+2^{-2 Q} l\right) n}=\prod_{r=0}^{2 Q-1}\left(1-e^{2 \pi i\left(\alpha+2^{-2 Q} l\right) 2^{r}}\right)
$$

и леммы 2 следует, что

$$
\left|\sum_{n \leqslant X} \varepsilon(n) e^{2 \pi i \alpha n}\right| \ll 2^{-2 Q} X^{\lambda} \sum_{l=1}^{2^{2 Q}} \min \left(X,\left\|2^{-2 Q} l\right\|^{-1}\right) .
$$

Отсюда и из леммы 1 вытекает утверждение следствия.

ЛЕмма 3 (П.К. Галлахер). Пусть $S(t)$ - комплекснозначная непрерывно дифберениируемая на отрезке $\left[t_{0}, t_{k}\right]$ функиия, $t_{0}<t_{1}<\cdots<t_{k-1}<t_{k}$. Тогда, полагая $\delta=\min _{0 \leqslant r<k}\left(t_{r+1}-t_{r}\right)$, имеем

$$
\sum_{r=1}^{k}\left|S\left(t_{r}\right)\right| \leqslant \frac{1}{\delta} \int_{t_{0}}^{t_{k}}|S(t)| d t+\frac{1}{2} \int_{t_{0}}^{t_{k}}\left|S^{\prime}(t)\right| d t .
$$

ДокАЗАТЕЛЬСтво см. в [6, гл. 1].

\section{§ 3. Основная лемма и следствия из нее}

Лемма 4. Пусть $Q \in \mathbb{N}, u$ nусть

$$
S_{Q}(a)=\prod_{r=0}^{2 Q-1}\left(1-e^{2 \pi i \alpha 2^{r}}\right)
$$

Справедливо неравенство

$$
\int_{0}^{1}\left|S_{Q}(\alpha)\right| d \alpha \leqslant 2^{Q \theta_{0}}
$$

где $\theta_{0}=\log _{2} \sqrt{2+\sqrt{2}}=0.88577 \ldots$.

ДоказАТЕЛЬСтво см. в [2].

СлеДСтвиЕ 2. Справедливо неравенство

$$
\int_{0}^{1}\left|\sum_{n \leqslant X} \varepsilon(n) e^{2 \pi i \alpha n}\right| d \alpha \leqslant X^{\theta_{0} / 2} \ln X .
$$

ДоказАтельство. Пусть $Q \in \mathbb{N}, 2^{2(Q-1)}<X+1 \leqslant 2^{2 Q}$. Тогда

$$
\int_{0}^{1}\left|\sum_{n \leqslant X} \varepsilon(n) e^{2 \pi i \alpha n}\right| d \alpha \ll 2^{-2 Q} \sum_{l=1}^{2^{2 Q}} \min \left(X,\left\|2^{-2 Q} l\right\|^{-1}\right) \int_{0}^{1}\left|S_{Q}\left(\alpha+2^{-2 Q} l\right)\right| d \alpha .
$$

Поскольку $S_{Q}(\alpha)$ - периодическая функция от $t$ с периодом 1 , имеем

$$
\int_{0}^{1}\left|S_{Q}\left(\alpha+2^{-2 Q} l\right)\right| d \alpha=\int_{0}^{1}\left|S_{Q}(\alpha)\right| d \alpha .
$$

В силу леммы 1 получаем

$$
2^{-2 Q} \sum_{l=1}^{2^{2 Q}} \min \left(X,\left\|2^{-2 Q} l\right\|^{-1}\right) \ll \ln X .
$$

Теперь утверждение следствия вытекает из леммы 4. 
СлеДСтвиЕ 3. Пусть $k \in \mathbb{N}$. Справедлива оченка

$$
2^{-k} \sum_{r=0}^{2^{k}-1}\left|\sum_{x=0}^{2^{k}-1} \varepsilon(x) e^{2 \pi i r x 2^{-k}}\right| \ll 2^{\theta_{0} k} k .
$$

ДокАЗАТЕЛЬСТво. Применяем лемму 3, положив в ней

$$
S(t)=\sum_{x=0}^{2^{k}-1} \varepsilon(x) e^{2 \pi i t x}, \quad t_{r}=\frac{r}{2^{k}} .
$$

Тогда

$$
S^{\prime}(t)=2 \pi i \sum_{x=0}^{2^{k}-1} x \varepsilon(x) e^{2 \pi i t x}
$$

Следовательно,

$$
2^{-k} \sum_{r=0}^{2^{k}-1}\left|\sum_{x=0}^{2^{k}-1} \varepsilon(x) e^{2 \pi i r x 2^{-k}}\right|=2^{-k} \sum_{r=0}^{2^{k}-1}\left|S\left(t_{r}\right)\right| \leqslant \int_{0}^{1}|S(t)| d t+2^{-k} \int_{0}^{1}\left|S^{\prime}(t)\right| d t .
$$

Применим к $S^{\prime}(t)$ преобразование Абеля:

$$
\begin{aligned}
2^{-k} \int_{0}^{1}\left|S^{\prime}(t)\right| & d t \ll 2^{-k} \int_{0}^{1} 2^{k}\left|\sum_{x=0}^{2^{k}-1} \varepsilon(x) e^{2 \pi i x t}\right| d t \\
+ & 2^{-k} \int_{0}^{1} \int_{0}^{2^{k}-1}\left(\left|\sum_{x \leqslant u} \varepsilon(x) e^{2 \pi i x t}\right| d t\right) d u \ll \int_{0}^{1}\left|\sum_{x \leqslant u_{0}} \varepsilon(x) e^{2 \pi i x t}\right| d t,
\end{aligned}
$$

где $u_{0}$ - то число из промежутка $\left[0,2^{k}-1\right]$, при котором последний интеграл максимален. Теперь утверждение следствия вытекает из следствия 2.

СлеДСтвиЕ 4. Пусть $k u t-$ целье числа, $0 \leqslant t \leqslant k$. Пусть при $m \in \mathbb{N}$

$$
\widehat{\varepsilon}_{m}(r)=2^{-m} \sum_{x=0}^{2^{m}-1} \varepsilon(x) e^{-2 \pi i r x 2^{-m}},
$$

и пусть а - произвольное число из промежутка $\left[0,2^{k}-1\right]$. Тогда

$$
\sum_{\substack{r=0 \\ r \equiv a\left(\bmod 2^{t}\right)}}^{2^{k}-1}\left|\widehat{\varepsilon}_{k}(r)\right| \ll 2^{(0.5-c)(k-t)}\left|\widehat{\varepsilon}_{t}(a)\right| k,
$$

где $c=\frac{1}{2}-\frac{\theta_{0}}{2}, \theta_{0}-$ константа из леммы 4.

ДокАЗАТЕЛЬСтво. По определению имеем

$$
\begin{aligned}
& \sum_{\substack{r=0 \\
r \equiv a\left(\bmod 2^{t}\right)}}^{2^{k}-1}\left|\widehat{\varepsilon}_{k}(r)\right|=2^{-k} \sum_{r \bmod 2^{k-t}}\left|\sum_{x=0}^{2^{k}-1} \varepsilon(x) e^{2 \pi i\left(a+2^{t} r\right) 2^{-k} x}\right| \\
& =2^{-k} \sum_{r \bmod 2^{k-t}}\left|\sum_{x=0}^{2^{k-t}-1} \sum_{y=0}^{2^{t}-1} \varepsilon\left(x+2^{k-t} y\right) e^{2 \pi i\left(a+2^{t} r\right) 2^{-k}\left(x+2^{k-t} y\right)}\right| .
\end{aligned}
$$


Поскольку $\varepsilon\left(x+2^{k-t} y\right)=\varepsilon(x) \varepsilon(y)$, имеем

$$
\begin{aligned}
\sum_{\substack{r=0 \\
r \equiv a\left(\bmod 2^{t}\right)}}^{2^{k}-1}\left|\widehat{\varepsilon}_{k}(r)\right| & =2^{-(k-t)} \sum_{r \bmod 2^{k-t}}\left|\sum_{x=0}^{2^{k-t}-1} \varepsilon(x) e^{2 \pi i\left(\frac{r}{2^{k-t}}+\frac{a}{2^{k}}\right) x}\right|\left|2^{-t} \sum_{y=0}^{2^{t}-1} \varepsilon(y) e^{2 \pi i \frac{a y}{2^{t}}}\right| \\
& =\left.2^{-(k-t)} \sum_{r \bmod 2^{k-t}}\right|^{2^{k-t}-1} \sum_{x=0} \varepsilon(x) e^{2 \pi i\left(\frac{r}{2^{k-t}}+\frac{a}{2^{k}}\right) x}|| \widehat{\varepsilon}_{t}(a) \mid .
\end{aligned}
$$

Сумма в правой части последнего равенства оценивается точно так же, как аналогичная сумма из следствия 3 .

\section{§4. Доказательство теоремы 1}

Воспользуемся тождеством Вона (см., например, [5, гл. 3, задача 9]), положив в нем $u=X^{0.1}$ :

$$
S=\sum_{n \leqslant X} \Lambda(n) \varepsilon(n)=W_{1}-W_{2}-W_{3}+O(u \ln u),
$$

где

$$
\begin{gathered}
W_{1}=\sum_{d \leqslant u} \mu(d) \sum_{n \leqslant X d^{-1}} \varepsilon(d n) e^{2 \pi i \alpha d n} \ln n \\
W_{2}=\sum_{d \leqslant u} \mu(d) \sum_{n \leqslant u} \Lambda(n) \sum_{d n r \leqslant X} \varepsilon(d n r) e^{2 \pi i \alpha d n r}, \\
W_{3}=\sum_{u<m \leqslant X u^{-1}} a_{m} \sum_{u<n \leqslant X m^{-1}} \Lambda(n) \varepsilon(m n) e^{2 \pi i \alpha m n}, \\
a_{m}=\sum_{d \mid m, d \leqslant u} \mu(d) .
\end{gathered}
$$

Суммы $W_{1}$ и $W_{2}$ оцениваются одинаково. Оценим, например, $W_{1}$.

Зафиксируем $d \leqslant u$. Применим к внутренней сумме, которую обозначим $S_{1}(d)$, преобразование Абеля; получим

$$
\left|S_{1}(d)\right| \ll\left|\sum_{d n \leqslant u_{0}} \varepsilon(d n) e^{2 \pi i \alpha d n}\right| \ln X,
$$

где $u_{0}$ - некоторое число, не превосходящее $X$.

Далее,

$$
\begin{gathered}
\sum_{d n \leqslant u_{0}} \varepsilon(d n) e^{2 \pi i \alpha d n}=\sum_{m \leqslant u_{0}} \varepsilon(m) e^{2 \pi i \alpha m} \frac{1}{d} \sum_{b=0}^{d-1} e^{2 \pi i \frac{b m}{d}}, \\
\left|\sum_{d n \leqslant u_{0}} \varepsilon(d n) e^{2 \pi i \alpha d n}\right| \leqslant \frac{1}{d} \sum_{b=0}^{d-1}\left|\sum_{m \leqslant u_{0}} \varepsilon(m) e^{2 \pi i\left(\alpha+\frac{b}{d}\right) m}\right| .
\end{gathered}
$$

Сумму по $m$ оценим по следствию 1:

$$
\left|\sum_{m \leqslant u_{0}} \varepsilon(m) e^{2 \pi i\left(\alpha+\frac{b}{d}\right) m}\right| \ll X^{\lambda} \ln X
$$


где $\lambda=0.792 \ldots$. Таким образом, для любых $d \leqslant u$ имеем

$$
\left|S_{1}(d)\right| \ll X^{\lambda} \ln X,
$$

следовательно,

$$
\left|W_{1}\right| \ll u X^{\lambda} \ln X
$$

Аналогично приходим к оценке

$$
\left|W_{2}\right| \ll u^{2} X^{\lambda} \ln X .
$$

Параметр $u$ выбран так, что

$$
\left|W_{1}\right| \ll X^{1-\varkappa_{1}}, \quad\left|W_{2}\right| \ll X^{1-\varkappa_{1}},
$$

где $\varkappa_{1}>0$ - абсолютная постоянная.

Оценим сумму $W_{3}$. Разобьем промежуток суммирования по $m$ на $O(\ln X)$ промежутков вида $\left(\frac{M}{2}, M\right]$, где $u<\frac{M}{2}<M \leqslant \frac{X}{u}$; один из таких промежутков может оказаться неполным. Имеем

$$
W_{3}=\sum_{M} W_{3}(M)
$$

где

$$
W_{3}(M)=\sum_{M / 2<m \leqslant M_{1}} a_{m} \sum_{u<n \leqslant X / m} \Lambda(n) \varepsilon(m n) e^{2 \pi i \alpha m n} ;
$$

здесь $\frac{M}{2}<M_{1} \leqslant M$.

Далее,

$$
\begin{aligned}
W_{3}(M)= & \sum_{M / 2<m \leqslant M_{1}} a_{m} \sum_{u<n \leqslant X / M_{1}} \Lambda(n) \varepsilon(m n) e^{2 \pi i \alpha m n} \\
& +\sum_{M / 2<m \leqslant M_{1}} a_{m} \sum_{X / M_{1}<n \leqslant X / m} \Lambda(n) \varepsilon(m n) e^{2 \pi i \alpha m n} .
\end{aligned}
$$

Разбивая промежуток суммирования по $n$ на $O(\log X)$ промежутков вида $\left(\frac{N}{2}, N_{1}\right]$, где $\frac{N}{2}<N_{1} \leqslant N, u<N \leqslant \frac{X}{M_{1}}$, приходим к неравенству

$$
\left|W_{3}\right| \ll\left|W_{3}(M, N)\right| \ln ^{2} X,
$$

где

$$
W_{3}(M, N)=\sum_{M / 2<m \leqslant M_{1}} a_{m} \sum_{N / 2<n \leqslant N_{1}} \Lambda(n) \varepsilon(m n) e^{2 \pi i \alpha m n}
$$

здесь $u<\frac{M}{2}<M_{1} \leqslant M \leqslant X u^{-1}, u<\frac{N}{2}<N_{1} \leqslant N \leqslant X u^{-1}$; может оказаться, что $N_{1}=X m^{-1}$.

Без ограничения общности считаем, что $M \leqslant N$.

Пользуясь тем, что $\left|a_{m}\right| \leqslant \tau(m) \ll m^{\varepsilon}$, и применяя неравенство Коши, получим

$$
\left|W_{3}(M, N)\right|^{2} \ll M^{1+\varepsilon} \sum_{M / 2<m \leqslant M_{1}}\left|\sum_{N / 2<n \leqslant N_{1}} \Lambda(n) \varepsilon(m n) e^{2 \pi i \alpha m n}\right|^{2} .
$$


Пусть $H=\left[X^{\rho}\right]$, где $0<\rho<10^{-3}$ - малый параметр, который будет выбран позже. Применим неравенство ван дер Корпута (см., например, [4], [5, гл. 1]):

$$
\begin{aligned}
\left|\sum_{N / 2<n \leqslant N_{1}} \Lambda(n) \varepsilon(m n) e^{2 \pi i \alpha m n}\right|^{2} \ll \frac{N}{H} \sum_{|h| \leqslant H}\left(1-\frac{|h|}{H}\right) \\
\times \sum_{\substack{N / 2<n \leqslant N_{1} \\
N / 2<n+h \leqslant N_{1}}} \Lambda(n) \Lambda(n+h) \varepsilon(m n) \varepsilon(m n+m h) e^{2 \pi i \alpha m n} e^{-2 \pi i \alpha m(n+h)} .
\end{aligned}
$$

Вклад $h=0$ оценивается как $O\left(\frac{N^{2}}{H}\right)$, поэтому

$$
\left|W_{3}(M, N)\right|^{2} \ll \frac{X^{1+\varepsilon}}{H} \sum_{h=1}^{H} \sum_{N / 2<n \leqslant N_{1}}\left|\sum_{M / 2<m \leqslant M_{1}} \varepsilon(m n) \varepsilon(m n+m h) e^{-2 \pi i \alpha m h}\right|+\frac{X^{2+\varepsilon}}{H} .
$$

Зафиксируем $h \in[1, H]$. Теперь достаточно доказать, что

$$
W_{4}(M, N)=\sum_{N / 2<n \leqslant N}\left|\sum_{M / 2<m \leqslant M_{1}} \varepsilon(m n) \varepsilon(m n+m h) e^{-2 \pi i \alpha m h}\right| \ll X^{1-\varkappa} .
$$

Выберем натуральное число $k$ из неравенств

$$
2^{k-1}<M X^{2 \rho} \leqslant 2^{k} .
$$

Введем функцию

$$
\varepsilon_{k}(n)=\left\{\begin{aligned}
1, & \text { если сумма первых } k \text { двоичных цифр } n \text { четна, } \\
-1 & \text { в противном случае. }
\end{aligned}\right.
$$

Докажем, что тогда

$$
\left|W_{4}(M, N)\right| \ll \sum_{N / 2<n \leqslant N}\left|\sum_{M / 2<m \leqslant M_{1}} \varepsilon_{k}(m n) \varepsilon_{k}(m n+m h) e^{-2 \pi i \alpha m h}\right|+X^{1-\rho+\varepsilon} .
$$

Разделим $m n$ на $2^{k}$ с остатком: $m n=2^{k} q+r, 0 \leqslant r<2^{k}$. Если $r<2^{k}-2 M H$, то $m n+m h=2^{k} q+r+m h, 0<r+m h<2^{k}$. Для таких $m n$ имеем

$$
\varepsilon(m n) \varepsilon(m n+m h)=\varepsilon(r) \varepsilon(r+m h)=\varepsilon_{k}(m n) \varepsilon_{k}(m n+m h) .
$$

Число пар $(m, n)$ таких, что остаток от деления $m n$ на $2^{k}$ лежит между $2^{k}-2 M H$ и $2^{k}-1$, есть $O\left(2^{-k} X M H X^{\varepsilon}\right)=O\left(X^{1-\rho+\varepsilon}\right)$.

Перейдем к оценке

$$
\left|W_{5}(M, N)\right|=\sum_{N / 2<n \leqslant N}\left|\sum_{M / 2<m \leqslant M_{1}} \varepsilon_{k}(m n) \varepsilon_{k}(m n+m h) e^{-2 \pi i \alpha h m}\right| .
$$

Введем для $\varepsilon_{k}(r)$ дискретное преобразование Фурье

$$
\widehat{\varepsilon}_{k}(r)=2^{-k} \sum_{l=0}^{2^{k}-1} \varepsilon_{k}(l) e^{-2 \pi i \frac{r l}{2^{k}}} .
$$


Из этого определения следует, что

$$
\begin{aligned}
\varepsilon_{k}(m n) & =\sum_{r=0}^{2^{k}-1} \widehat{\varepsilon}_{k}(r) \exp \left\{\frac{2 \pi i r m n}{2^{k}}\right\}, \\
\varepsilon_{k}(m n+m h) & =\sum_{s=0}^{2^{k}-1} \widehat{\varepsilon}_{k}(s) \exp \left\{\frac{2 \pi i s(m n+m h)}{2^{k}}\right\} .
\end{aligned}
$$

Суммируя получившиеся линейные суммы по $m$, получаем неравенство

$$
\left|W_{5}(M, N)\right| \leqslant \sum_{r=0}^{2^{k}-1} \sum_{s=0}^{2^{k}-1}\left|\widehat{\varepsilon}_{k}(r)\right|\left|\widehat{\varepsilon}_{k}(s)\right| \sum_{N / 2<n \leqslant N} \min \left(M,\left\|\frac{r+s}{2^{k}} n+\frac{s h}{2^{k}}-\alpha h\right\|^{-1}\right) .
$$

Далее будем считать, что $\rho=\frac{c}{200}$, где $c=\frac{1-\theta_{0}}{2}, \theta_{0}-$ константа из леммы 4 .

Пусть $t$ - целое неотрицательное число такое, что $2^{t} \|(r+s)$, последнее означает, что $2^{t} \mid(r+s)$, но $2^{t+1} \nmid(r+s)$.

Пусть сначала $0 \leqslant t \leqslant k-\frac{2 \rho}{c} \log _{2} X$. Заметим, что из неравенств $2^{k}>M \geqslant$ $X^{1 / 10}$ следует, что $k>\frac{1}{10} \log _{2} X$; отсюда и из равенства $\frac{2 \rho}{c}=\frac{1}{100}$ вытекает неравенство $k-\frac{2 \rho}{c} \log _{2} X \geqslant \frac{4}{5} k$.

Применим к сумме

$$
\sum_{N / 2<n \leqslant N} \min \left(M,\left\|\frac{r+s}{2^{k}} n+\frac{s h}{2^{k}}-\alpha h\right\|^{-1}\right)
$$

лемму 1 , положив в ней $q=2^{k-t}, \alpha=\frac{r+s}{2^{k}}, \beta=\frac{s h}{2^{k}}-\alpha h$; получим

$$
\sum_{N / 2<n \leqslant N} \min \left(M,\left\|\frac{r+s}{2^{k}} n+\frac{s h}{2^{k}}-\alpha h\right\|^{-1}\right) \ll\left(\frac{N}{2^{k-t}}+1\right)\left(M+2^{k-t} k\right) .
$$

Упростим правую часть последнего неравенства. Имеем

$$
\begin{gathered}
\frac{N}{2^{k-t}}+1<\frac{N X^{2 \rho}}{2^{k-t}}+1 \leqslant 2 \frac{N X^{2 \rho}}{2^{k-t}}, \\
M+2^{k-t} k<M X^{2 \rho}+2^{k-t} k<2 M X^{3 \rho},
\end{gathered}
$$

таким образом,

$$
\begin{gathered}
\left(\frac{N}{2^{k-t}}+1\right)\left(M+2^{k-t} k\right)<4 \cdot 2^{-(k-t)} M N X^{5 \rho} \leqslant 5 \cdot 2^{-(k-t)} X^{1+5 \rho}, \\
\sum_{N / 2<n \leqslant N} \min \left(M,\left\|\frac{r+s}{2^{k}} n+\frac{s h}{2^{k}}-\alpha h\right\|^{-1}\right) \ll 2^{-(k-t)} X^{1+5 \rho} .
\end{gathered}
$$

Теперь оценим сумму

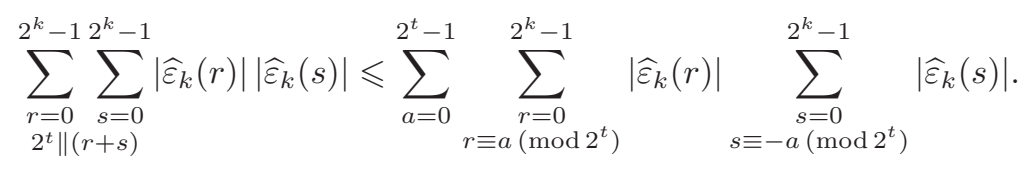


Воспользуемся следствием 4:

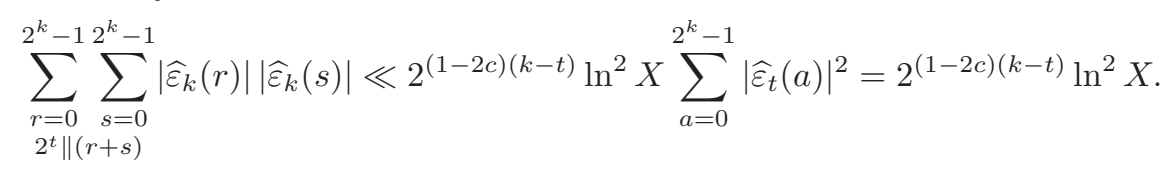

В рассматриваемом случае получена оценка

$$
\left|W_{5}(M, N)\right| \ll 2^{-2 c(k-t)} X^{1+6 \rho} \ln ^{2} X .
$$

Учитывая, что

$$
k-t \geqslant \frac{4}{5} k, \quad 2^{k}>M \geqslant X^{1 / 10},
$$

получаем

$$
\left|W_{5}(M, N)\right| \ll X^{1+6 \rho-\frac{8}{50} c} ;
$$

наконец, из неравенства $\rho \leqslant \frac{c}{100}$ имеем $6 \rho-\frac{8}{50} c<-\rho$,

$$
\left|W_{5}(M, N)\right| \ll X^{1-\rho} .
$$

Осталось рассмотреть случай

$$
k-\frac{2 \rho}{c} \log _{2} X<t \leqslant k .
$$

Имеем

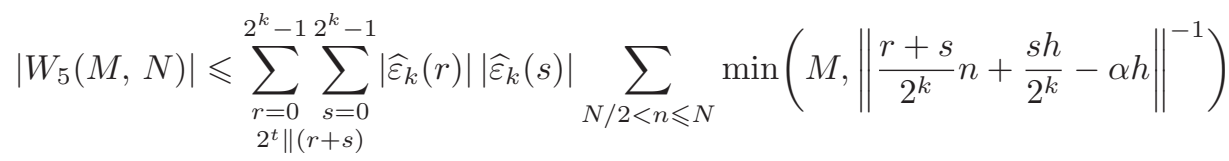

$$
\begin{aligned}
& =\sum_{N / 2<n \leqslant N} \sum_{s_{2}=0}^{2^{k-t}-1} \sum_{r_{2}=0}^{2^{k-t}-1} \sum_{\substack{s_{1}=0 \\
r_{1}+s_{1} \equiv 0\left(\bmod 2^{t}\right)}}^{2^{t}-1} \sum_{r_{1}=0}^{2^{t}-1}\left|\widehat{\varepsilon}_{k}\left(r_{1}+2^{t} r_{2}\right)\right|\left|\widehat{\varepsilon}_{k}\left(s_{1}+2^{t} s_{2}\right)\right| \\
& \times \min \left(M,\left\|\frac{r_{1}+s_{1}+2^{t}\left(r_{2}+s_{2}\right)}{2^{k}} n+\frac{\left(s_{1}+2^{t} s_{2}\right) h}{2^{k}}-\alpha h\right\|^{-1}\right) .
\end{aligned}
$$

При $0 \leqslant s_{1}, r_{1}<2^{t}$ из сравнения $r_{1}+s_{1} \equiv 0\left(\bmod 2^{t}\right)$ следует, что либо $r_{1}=s_{1}=0$, либо $r_{1}+s_{1}=2^{t}$. Отсюда и из леммы 2 получаем

$$
\begin{array}{r}
\left|W_{5}(M, N)\right| \ll 2^{-2 k(1-\lambda)} \sum_{N / 2<n \leqslant N} \sum_{s_{2}=0}^{2^{k-t}-1} \sum_{r_{2}=0} \sum_{s_{1}=0}^{2^{k-t}-1} \min \left(M,\left\|\frac{h s_{1}}{2^{k}}+\beta\right\|^{-1}\right) \\
+2^{-2 k(1-\lambda)} 2^{2(k-t)} X
\end{array}
$$

где $\beta=\frac{1+r_{2}+s_{2}}{2^{k-t}} n+\frac{h s_{2}}{2^{k-t}}-\alpha h$.

Пусть $\frac{h s_{1}}{2^{k}}=\frac{h_{1} s_{1}}{2^{k_{1}}}$, где $\frac{h_{1} s_{1}}{2^{k_{1}}}-$ несократимая дробь. В силу леммы 1 и неравенств

$$
2^{-k_{1}} \leqslant 2^{-k} X^{\rho}, \quad M+2^{k_{1}} k_{1} \ll M X^{3 \rho}
$$

имеем

$$
\sum_{s_{1}=0}^{2^{t}-1} \min \left(M,\left\|\frac{h s_{1}}{2^{k}}+\beta\right\|^{-1}\right) \ll M X^{5 \rho} .
$$

Подставляя неравенство (2) в соотношение (1), получаем

$$
\left|W_{5}(M, N)\right| \ll 2^{k-t} 2^{-2 k(1-\lambda)} X^{1+5 \rho} .
$$


Теперь воспользуемся тем, что

$$
2^{k-t} \leqslant X^{4 \rho / c}, \quad \frac{\rho}{c} \leqslant \frac{1}{200}, \quad c<0.06, \quad 2^{k}>M \geqslant X^{0.1} .
$$

Получим

$$
\left|W_{5}(M, N)\right| \ll X^{1-0.01} .
$$

Теорема 1 доказана.

\section{§5. Доказательство теоремы 2}

Пусть

$$
S(\alpha)=\sum_{p \leqslant N} e^{2 \pi i \alpha p}, \quad S_{0}(\alpha)=\sum_{p \leqslant N} \varepsilon(p) e^{2 \pi i \alpha p} .
$$

Тогда

$$
J_{0}(N)=\frac{1}{8} \int_{0}^{1}\left(S(\alpha)+S_{0}(\alpha)\right)^{3} e^{-2 \pi i \alpha N} d \alpha .
$$

Раскрывая скобки и пользуясь теоремой 1 и неравенством Коши, получаем

$$
J_{0}(N)=\frac{1}{8} \int_{0}^{1} S^{3}(\alpha) e^{-2 \pi i \alpha N} d \alpha+O\left(\pi(N) N^{1-\varkappa}\right) .
$$

Поскольку

$$
J(N)=\int_{0}^{1} S^{3}(\alpha) e^{-2 \pi i N \alpha} d \alpha, \quad J(N) \gg N^{2}(\ln N)^{-3}
$$

(при достаточно больших нечетных $N$ ), теорема 2 доказана.

\section{Список литературы}

1. A. O. Gel'fond, "Sur les nombres qui ont des propriétés additives et multiplicatives données", Acta Arith., 13 (1968), 259-265.

2. К. М. Эминян, "О проблеме делителей Дирихле в некоторых последовательностях натуральных чисел”, Изв. АН СССР. Сер. матем., 55:3 (1991), 680-686; англ. пер.: K. M. Ėminyan, "On the Dirichlet divisor problem in some sequences of natural numbers", Math. USSR-Izv., 38:3 (1992), 669-675.

3. C. Mauduit, J. Rivat, "Sur un probléme de Gelfond: la somme des chiffres des nombres premiers", Ann. of Math. (2), 171:3 (2010), 1591-1646.

4. B. Green, Three topics in additive prime number theory, arXiv: 0710.0823.

5. А.А. Карацуба, Основы аналитической теории чисел, Наука, М., 1983; англ. пер.: A. Karatsuba, Basic analytic number theory, Springer-Verlag, Berlin, 1993.

6. Г. Монтгомери, Мультипликативная теория чисел, Мир, М., 1974; пер. с англ.: H. L. Montgomery, Topics in multiplicative number theory, Lecture Notes in Math., 227, Springer-Verlag, Berlin, 1971.

КАРАПЕТ МКРТИЧЕВИЧ ЭМИНЯН

Поступило в редакцию

(KARAPET M. EMINYAN)

10.04 .2012

Финансовый университет при Правительстве РФ,

г. Москва;

Московский государственный технический

университет им. Н.Э. Баумана

E-mail: eminyan@mail.ru 